\title{
Effets de l'avotan sur les flux intestinaux de l'azote chez la vache laitière : étude préliminaire
}

\author{
D Ali-Haimoud, C Bayourthe \\ École nationale supérieure agronomique, laboratoire d'Ingénierie agronomique, 145, avenue de Muret, \\ 31076 Toulouse Cedex, France
}

L'avotan, antibiotique actif sur les bactéries Gram+, est utilisé comme additif dans l'alimentation des volailles, du porc et des bovins à l'engrais. L'objectif de cette étude a été de rechercher les éventuelles conséquences d'un apport d'avotan sur les flux intestinaux d'azote $(N)$, chez la vache laitière.

Quatre vaches Holstein canulées (rumen, duodénum, iléum) ont reçu une ration composée (kg MS) d'ensilage de maïs plante entière $(4,4)$, de foin de ray-grass $(3,7)$ et d'un concentré azoté $(1,8)$; l'avotan a été incorporé dans l'aliment à raison de $100 \mathrm{mg}$. Le $\mathrm{Cr}$-EDTA, $\mathrm{YbCl}_{3}$ et ${ }^{15} \mathrm{~N}$ ont été utilisés comme marqueurs respectifs des phases liquide et solide et des protéines bactériennes; les flux ont été calculés selon la méthode de Faichney (1980).

L'adjonction d'avotan à la ration n'a pas influencé la digestibilité totale de $N(74 \%)$. Dans le rumen, la dégradation apparente des matières azotées a baissé (53 vs
$63 \%$ ), parallèlement le flux $N$ duodénal alimentaire a augmenté de $22 \%$, le flux $\mathrm{N}$ bactérien étant inchangé (tableau I). Le flux $N$ iléal alimentaire n'a pas varié, la quantité de $\mathrm{N}$ absorbée dans l'intestin, de même que l'efficacité de cette absorption ont été plus élevées, soit respectivement: 22 vs $15 \%$ ingéré et 48 vs $40 \%$ entrées. Malgré le peu d'animaux utilisés, il est toutefois possible d'avancer que l'avoparcine $(100 \mathrm{mg} / \mathrm{j})$ réduit la dégradation des matières azotées dans le rumen et accroît la fraction azotée d'origine alimentaire, qui est apparemment absorbée dans l'intestin.

Remerciements - Ce travail a été soutenu par la société Cyanamid (74, rue d'Arcueil, 94578 Rungis Cedex, France).

Faichney GJ (1980) Austr J Agric Res 31, 11291137

Tableau I. Influence de l'avoparcine $(100 \mathrm{mg} / \mathrm{j})$ sur les flux $(\mathrm{g} / \mathrm{j})$ azotés intestinaux chez la vache laitière.

$N$ ingéré $(g / j)$

$\mathrm{N}$ duodénal alimentaire + endogène

$\mathrm{N}$ duodénal bactérien

$\mathrm{N}$ iléal alimentaire + endogène

$N$ fécal

$\begin{array}{rr}249,3 \pm 12,5 & 241,4 \pm 4,3 \\ 93,1 \pm 0,2 & 113,3 \pm 12,2 \\ 89,9 \pm 87,0 & 91,6 \pm 3,7 \\ 55,6 \pm 3,8 & 59,4 \pm 1,2 \\ 60,8 \pm 2,2 & 63,2 \pm 0,6\end{array}$

